on the risks of future children being affected the specific genetic syndromes should first be considered. The recurrence risk will be that for the syndrome. In cases due to progestin or sex chromosome anomalies the risk for future offspring will usually be small. For the rest, the empirical risk for later brothers of patients born to unaffected fathers may be taken to be about $10 \%$.

1 Sørensen, H. R., Hypospadias with Special Reference to Aetiology. Copenhagen, Munksgaard, 1953

- Lamy, M., Lecture read at the University Institute of Human Genetics, Copenhagen, 18 April 1952.

Aarskog, D., Acta Paediatrica Scandinavica, 1970, Supplement 203.

- Chen, Y. C., and Woolley, P. V., Fournal of Medical Genetics, 1971, 8, 153.

\section{Tests on the Pill for Carcinogenicity}

This week the Committee on Safety of Medicines published its long-awaited report on the tests of various oral contraceptives for carcinogenicity in rats and mice. ${ }^{1}$ Taken at their face value the conclusions drawn by the committee are reassuring. Each oral contraceptive preparation was tested on male and female animals of both species at three dose levels-a low dose (2-5 times the human contraceptive dose), a medium dose (50-150 times), and a high dose (200400 times the human contraceptive dose). The experiments on mice lasted 80 weeks and those on rats two years. Animals exposed to medium and high doses of many of the compounds developed more pituitary (rats and mice) and mammary tumours (rats only) than controls and animals receiving low doses, but this was only to be expected in view of the known effects of high doses of oestrogens on the risk of the development by susceptible strains of rats and mice of neoplasms of these types. An earlier report to the committee by $G$. Bonser had suggested that dosage with mestranol was associated with the development of liver damage, nodular hyperplasia, and hepatomas in rats. The studies now reported are regarded by the committee as not supporting her findings.

The main conclusion is that "although a carcinogenic effect can be produced when some of the preparations are used in high doses throughout the life-span in certain strains of rat and mouse, this evidence cannot be interpreted as constituting a carcinogenic hazard to women when these preparations are used as oral contraceptives." On the other hand the committee proposes to review the situation when the results of long-term studies on primates and beagle bitches, now in progress in the United States, become available. In the meantime it recommends careful monitoring of cancer incidence in women taking oral contraceptives and "careful documentation, investigation and follow-up of all cases of amenorrhoea following hormonal contraception" in view of the effects on the pituitary gland shown by the rat and mouse experiments.

It is interesting to compare the reaction of this committee with that of the Food and Drug Administration in the United States, which has recently banned DDT on the grounds that it increases the risk of liver tumours in mice. ${ }^{2}$ In the oral contraceptive studies male rats showed a pronounced, doserelated, increased risk of developing liver tumours in response to norethynodrel alone or together with mestranol $66: 1$ or to norethisterone alone or with mestranol. Megestrol acetate in combination with ethinyloestradiol and ethinyloestradiol alone increased the incidence of liver tumours in both male and female rats. Despite these findings the committee states boldy. "The extensive tests here reported do not support the previous work showing liver damage progressing to nodular hyperplasia and an increased incidence of hepatomas from prolonged administration of oral contraceptive preparations to rats." This is, strictly speaking, true insofar as no liver damage was encountered, but the statement sweeps a lot of liver tumours under the carpet.

The report is a masterpiece of brevity, compressing the findings of studies on over 13,000 animals into 15 pages and 7 tables, but the experimentalist used to scrutinizing data from long-term animal studies will note that some important information is missing. For example, the report states: "In some instances the high doses of the compounds led to premature death of the animals, either from general toxicity or from certain tumours. As a result, the incidence of other tumours may have been reduced. This needs to be borne in mind when assessing tumour yield." The last sentence is very true, but the reader of the report is left with a problem on his mind because data on early deaths are not given. Another important omission is any information on whether treatment of female animals with the compounds was associated with suppression of ovulation. If not, can there be any assurance that exposure reproduced the hormonal state of women taking the "pill"? If the risk of cancer is altered in either direction in women on the pill, the change in risk is likely to be attributable to interference with the delicate feedback mechanisms which control menstruation and ovulation. Massive exposure to hormones of species in which the control mechanisms are basically different is a priori unlikely to provide interpretable results.

Readers unfamiliar with laboratory rats and mice may well be surprised at the high incidences of some types of neoplasms found in untreated control animals. The tables in the report show incidences of $25 \%$ of lung tumours and $17 \%$ of liver tumours in control mice and $26 \%$ adrenal tumours, $30 \%$ pituitary tumours, and $99 \%$ mammary tumours in control rats. It is difficult to see how experiments on strains of animals so exceedingly liable to develop tumours of these various kinds can throw useful light on the carcinogenicity of any compound for man. Indeed the value of the mouse as a species for carcinogenicity testing has recently been seriously questioned because of a high incidence of tumours in untreated controls. ${ }^{3}$

Many people who feel oppressed by the increasing threat of world overpopulation would desperately like the "pill" to be found safe from the point of view of cancer. The studies now reported neither incriminate oral contraceptives as carcinogens nor exonerate them. We shall simply have to wait and see what the epidemiologists learn from prospective studies.

\footnotetext{
1 Committee on Safety of Medicines, Carcinogenicity Tests of Oral Contraceptives, London, H.M.S.O., 1972.

2 Nature, 1972, 237, 420 and 422.

3 Grasso, P., and Crampton, R. F., 1972, Fod and Cosmetics Toxicology, 10, 418.
}

\section{W.H.O. in Europe}

From its new building in Copenhagen the Regional Office for Europe of the World Health Organization is directing research, organizing measures to improve public health, and helping with educational schemes in the countries it covers. 